\title{
Correction to: Do customers exhibit gratitude after service recovery? Understanding the moderating role of relationship type
}

\author{
Ramakrishna Salagrama $^{1} \cdot$ Sanjeev Prashar $^{1} \cdot$ T. Sai Vijay ${ }^{2}[$
}

Published online: 10 November 2021

๑) Springer-Verlag GmbH Germany, part of Springer Nature 2021

\section{Correction to: Service Business https://doi.org/10.1007/s11628-021-00468-3}

Due to some communication oversight during article acceptance, first author Ramakrishna Salagrama was not correctly mentioned as Corresponding author.

This error has been corrected with original article updated.

Publisher's Note Springer Nature remains neutral with regard to jurisdictional claims in published maps and institutional affiliations.

The original article can be found online at https://doi.org/10.1007/s11628-021-00468-3.

Ramakrishna Salagrama

ramakrishnasalagrama@gmail.com

Sanjeev Prashar

dr.sanjeev.prashar@gmail.com

T. Sai Vijay

tsaivijay@gmail.com

1 Indian Institute of Management Raipur, Atal Nagar, P. O. - Kurru (Abhanpur) Village - Pota Cheria, Naya Raipur, Chhattisgarh, India

2 Indian Institute of Management Ranchi, Suchna Bhavan, 5th Floor, Audrey House Campus, Meur's Road, Ranchi 834008, Jharkhand, India 